\title{
Clinical Profile and Outcome of Steroid Resistant Nephrotic Syndrome in Children: An Eastern Indian Single Centre Study
}

\author{
Pawan Mutalik $^{1^{*}}$, Subal Kumar Pradhan ${ }^{2}$, Sumanta Panigrahi ${ }^{3}$, \\ Anil Kumar Mohanty ${ }^{4}$ \\ ${ }_{1,2,3,4}$ Sardar Vallabh Bhai Patel Post Graduate Institute of Pediatrics (SVPPGIP) and SCB Medical College, \\ Utkal University, Cuttack, Odisha, India
}

\begin{abstract}
:
Background: Steroid resistant Nephrotic syndrome (SRNS) is a therapeutic challenge for pediatricians and pediatric nephrologists worldwide. Currently, numerous immunosuppressive agents are used with variable efficacy.

Materials and Methods: This prospective study was conducted from February 2008 to October 2013 in the department of Pediatrics at SVPPGIP and SCB Medical College, Cuttack, India in 44 children with SRNS aged 6 months-14 years. The demographic, clinical, laboratory data at the time of presentation, response to various therapies and follow up data of 12 months was noted. Data was compiled and analyzed with Microsoft Excel software.

Results: Out of 44 SRNS children, Male: female ratio was 1.2:1. Nineteen (43.18\%) children had Minimal Change Disease (MCD), 13 (29.54\%) had Focal Segmental Glomerulosclerosis (FSGS), 7 (15.9\%) cases had IgA Nephropathy (IgAN) and 3 cases had IgM Nephropathy (IgMN). Most $(n=29 ; 65.9 \%)$ of the children responded to Cyclosporin A (CyA). Thirty seven (84.1\%) cases were late steroid responders. During follow up, $39(88.64 \%)$ children were in complete remission. There were no major adverse effects except Cushingoid features and Hirsutism.
\end{abstract}

Conclusions: In our study, MCD was most common histological subtype of SRNS and majority of the children responded to Cyclosporin A.

Keywords: Steroid resistance, Children, Cyclosporin A, Immunosuppressants, Minimal change disease, Nephrotic syndrome

\section{Introduction}

Idiopathic Nephrotic syndrome (INS) affects about 2 per 1 lakh children aged $\leq 15$ years [1,2]. About $10 \%$ to $20 \%$ of these children are resistant to steroids [1]. Response to steroids is associated with a good longterm prognosis [3]. Management of SRNS is difficult, most patients failing to achieve remission show progressive renal damage [4]. Steroid dependence or resistance seems to have increased over the last decades, but the treatment of difficult cases of INS, mostly due to FSGS, is still a challenge. A number of medications such as Methyl prednisolone, Cyclophosphamide, Vincristine, Mycophenolate Moefetil (MMF) and CyA have been used with varying results. Recent reports have showed remission rates ranging from $20 \%$ to $70 \%$ using these drugs in SRNS [5, 6, 7]. Moreover, there are some reports supporting the idea that MCD, Diffuse Mesangial Proliferation (DMP), and FSGS are separate diseases because of different response rate to corticosteroid and the histological findings offer data of prognostic significance [8]. Therefore, in many centers, renal biopsy is recommended according to the rationale that the intensity of immunosuppression may have to be adapted to the underlying findings in renal biopsy for the patients who do not respond to steroids $[8,9,10]$. Achievement of complete or partial remission with any therapy reduced the risk of End stage renal disease (ESRD) [11].

In this study, we tried to determine the clinical profile and outcome of childhood SRNS in our tertiary level pediatric referral centre. Literature is scanty on this topic throughout the world especially regarding the outcome of immunosuppressant therapy in childhood SRNS. Hence, this study was conducted to determine the varying outcomes of SRNS patients.

\section{Study Design And Methodology}

This descriptive and prospective study was conducted from February 2008 to October 2013 in the department of Pediatrics at SVPPGIP and SCB Medical College, Cuttack, Orissa, India. Total 44 children with SRNS aged between 6 months to 14 years were included in the study. As per the Indian Society of Pediatric Nephrology (ISPN) protocol, all these children were subjected to renal biopsy after taking informed written consent from parents or legal guardians and hence were exempted from ethical clearance. The demographic, 
clinical, laboratory data at the time of presentation, response to various therapies and follow up status were retrieved from case files. Definitions of the disease, treatment and its responses were in accordance with the ISPN consensus guideline as follows [4]:

Complete remission (CR): Urine albumin trace or nil or urine Protein Creatinine ratio $<0.2 \mathrm{mg} / \mathrm{mg}$ for 3 consecutive days.

Partial remission (PR): Urine albumin $1+$ or $2+$ or Urine Protein Creatinine ratio 0.2 to $2 \mathrm{mg} / \mathrm{mg}$.

Non-responder: No remission.

Relapses: Urine albumin $3+$ or $4+$ or protein creatinine ratio $\geq 2 \mathrm{mg} / \mathrm{mg}$ for 3 consecutive days.

SRNS: Lack of remission despite daily therapy with oral prednisolone at a dose of $2 \mathrm{mg} / \mathrm{kg} /$ day for 4 weeks.

Late responder: Initially resistant to steroids but later on responsive to it.

MCD: also known as lipoid nephrosis, this entity, on light microscopy examination demonstrates mild changes within the Glomerular mesangium consisting of either proliferation or sclerosis and without significant Immunofluorescence deposits. Most common sub-type in our study as shown in Figure 1

FSGS: It is a histopathological diagnosis characterized by sclerosis of focal (only some of the glomeruli) and segmental (part of the glomeruli) glomeruli and Immuno-Fluorescence (IF) is usually negative. Second most common sub-type in our study as shown in Figure 2

All this data was compiled and analyzed using Microsoft Excel software. The mean duration of follow up was 12 months.

\section{Results}

A clinical diagnosis of SRNS was made in 44 children. Twenty four (54.5\%) were males and 20 (45.5\%) were females with a male-to-female ratio of 1.2:1 as shown in Table I. The mean age was $4.6 \pm 3.2$ years (range 6 months-14 years). Four (9\%) out of these 44 were aged between 10-14 years. Out of 44 children, 19 (43.18\%) children had MCD, 13 (29.54\%) had FSGS, 7 (15.9\%) cases had nephrotic IgAN, 3 (6.81\%) cases had IgMN and one case was of primary Mesangial Proliferative Glomerulonephritis and primary Membranous Nephropathy each. Hypertension was present in $15(34 \%)$ and $27(61.3 \%)$ cases had hematuria (microscopic/clinical) with all children having normal kidney function test at the time of renal biopsy. All the children had received prednisolone before adding a second immunosuppressant. Out of 44 children, 28 children responded to CyA, 10 children responded to CYP, 4 children responded to oral Tacrolimus and remaining 2 cases went into remission with MMF as shown in Table II. The most common adverse effect in our study was Cushingoid features $(n=18)$. This was followed by steroid induced cataract $(n=5)$, Hirsutism $(n=3)$, Leucopenia $(\mathrm{n}=2)$ and creatinine $>150 \%$ of baseline $(\mathrm{n}=2)$. There were three deaths due to sepsis in our study, two of them had FSGS and one had MCD.

Follow -up: In our study, the mean duration for induction of remission for MCD cases was 80 days and for FSGS cases it was 115 days. Complete remission was achieved in 39 (84.1\%) cases, 4 (9.1\%) cases had partial remission and one case was a non-responder (died due to septicemia). Three (6.8\%) patients died during the course of study, all due to septicemia.

\section{Figure Legends}

Fig. 1: Renal biopsy of a 4 year old boy; light microscopy showing glomeruli within normal limits of morphology. Unremarkable blood vessels, Interstitium and tubules suggestive of Minimal change disease (Jones' silver stain; original magnification x200)

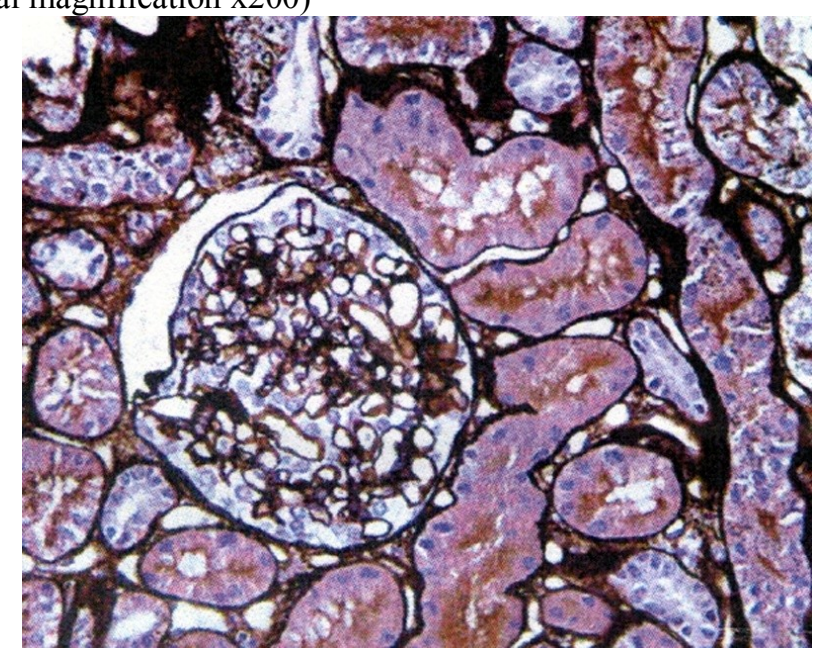


Fig. 2: Renal biopsy of an 11 year old girl; light microscopy showing glomeruli with segmental sclerosis, podocyte proliferations and adhesion to the capsule. Basement membrane not thickened, Interstitium shows mild to moderate diffuse lymphocytic infiltration, tubules show hyaline casts with blood vessels being unremarkable. Features suggestive of focal segmental glomerulosclerosis (H\&E stain; original magnification x200)

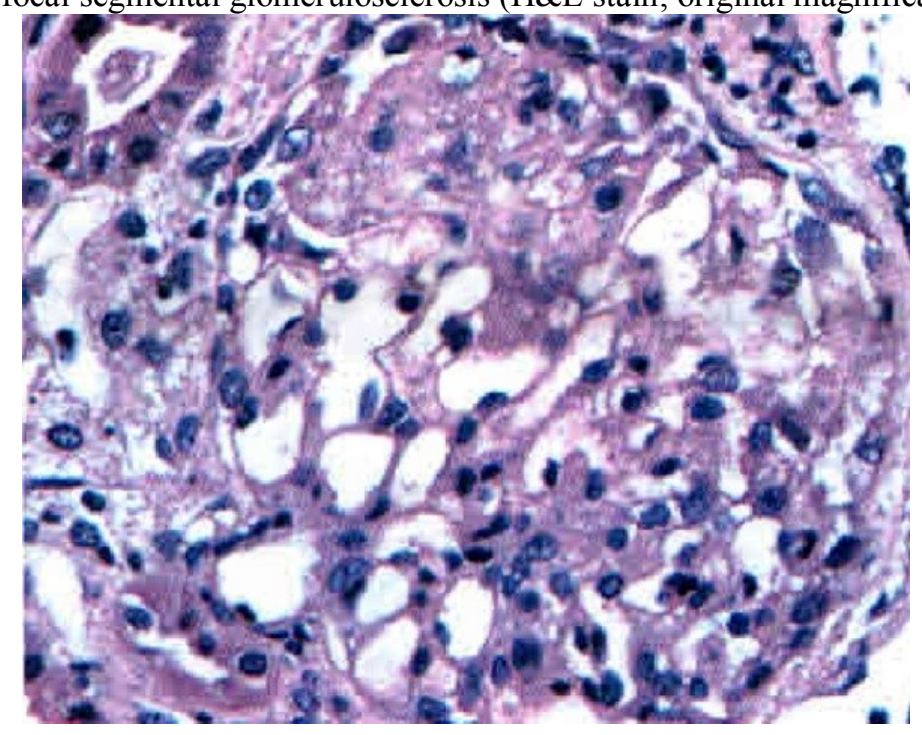

Details of staining:

Fixation: For light microscopy, buffered 10\% aqueous formaldehyde solution (formalin) was utilized.

Stains: For light microscopy, we have used Hematoxylin and Eosin (H\&E) stain and Jones' methenamine silver stain for Glomerular capillary basement membrane (GBM).

\section{Tables}

Table I: Profile of cases in our study $(n=44)$

\begin{tabular}{|ll|}
\hline Number of patients & 44 \\
\hline Male : Female & $1.2: 1$ \\
\hline Mean age of diagnosis & 4.6 years \\
\hline Mean time for remission & 76 days \\
\hline Number of MCD & $19(43.18 \%)$ \\
\hline Number of FSGS & $13(29.54 \%)$ \\
\hline Number of Deaths & 3 \\
\hline Number of Late responders & $37(84.1 \%)$ \\
\hline
\end{tabular}

*FSGS: Focal segmental glomerulosclerosis

${ }^{\#}$ MCD: Minimal change disease

\begin{tabular}{|c|c|c|c|c|c|c|}
\hline Drugs & Number & CR & PR" & $\mathrm{NR}^{\sim}$ & $\begin{array}{l}\text { Remission }^{2} \quad \text { with } \\
\text { drugs }(\%)\end{array}$ & $\begin{array}{l}\text { Number of adverse } \\
\text { events }\end{array}$ \\
\hline${ }^{1} \mathrm{CyA}$ & $29(65.9 \%)$ & 19 & 3 & 2 & 76 & 5 \\
\hline${ }^{2} \mathrm{Cyp}$ & $24(54.5 \%)$ & 10 & 0 & 14 & 58.3 & 2 \\
\hline MIIF & $2(4.5 \%)$ & 2 & 0 & 0 & 100 & 0 \\
\hline Tacrolimus & $4(9.1 \%)$ & 3 & 1 & 0 & 100 & 0 \\
\hline
\end{tabular}

Table II: Treatment response and adverse events of various Immuno-suppressants.

${ }^{a} \mathrm{CR}$ or PR at the end of treatment course with individual drugs.

*CR: Complete remission

${ }^{\#}$ PR: Partial remission

NR: No remission

${ }^{1}$ CyA: Cyclosporine-A

${ }^{2}$ CYP: Cyclophosphamide

${ }^{3}$ MMF: Mycophenolate moefetil 


\section{Discussion And Conclusion}

Steroid resistant Nephrotic syndrome patients have a very variable course with some developing chronic renal failure and other remaining persistently Nephrotic [2]. Achieving remission in children with SRNS is difficult and many immunosuppressive drugs have been used with variable success [18]. However, remission is achievable with cyclosporine and other immunosuppressive agents. Treatment should be individualized according to the underlying histopathology, clinical parameters and social conditions of the child [18]. In our study, maximum number of children responded to Cyclosporin A $(n=29)$ and four children received Tacrolimus. Tacrolimus is an effective therapeutic modality for SRNS, including the subgroup of children who are nonresponsive to the current therapeutic modalities like Cyclophosphamide and cyclosporine A [19]. MMF is a promising therapeutic alternative without nephrotoxicity [20]. But there is no single consensus regarding the indications of renal biopsy and treatment protocols in children with SRNS [13, 14, 15, 16]. Optimal combinations of these agents with the least toxicity remain to be determined [12]. Although the response to treatment has the best prognostic indicator in children with Nephrotic syndrome, renal biopsy has a definite prognostic value in SRNS $[18,19]$. Our study showed that MCD children had earlier remission with Immunosuppressants compared to FSGS cases ( 80 days v/s 115 days). In children, renal biopsy is routinely required in the management of idiopathic steroid-resistant Nephrotic syndrome particularly prior to starting immunesuppressive agents [21]. Most of the patient resistant to steroid therapy had good response to other immune suppressive therapies. Most of the patients achieved partial or complete remission within one year of follow up. Immunosuppressive agents are used to prolong periods of remission in children; however these agents have significant potential adverse effects [6]. However, in our study there were no major side effects. None of the adverse drug events resulted in significant morbidity and hence a favorable risk-benefit ratio of these drugs is present. However, a larger cohort with continued follow-up of the current cases is required to know the long term outcome of these patients.

\section{Limitations:}

1. Lack of an untreated control group.

2. A randomized trial will be needed to assess the true value of each drug in comparison to placebo. Such a study will be very difficult due to the presumed efficacy of Immuno-suppressants.

3. Blood drug levels could not be collected in the patients under the study and hence a toxicity dosage level couldn't be determined.

4. Degree of adherence to prescribed medications couldn't be evaluated in every case.

5. A longer period of follow up with repeated renal biopsies would have revealed other histopathological diagnoses and hence treatment outcomes probably.

None.

\section{Acknowledgements}

\section{References}

[1]. McKinney PA, Feltbower RG, Brocklebank JT, Fitzpatrick MM. Time trends and ethnic patterns of childhood Nephrotic syndrome in Yorkshire. UK Pediatr Nephrol; 2001: 16:1040-1044.PMID: 11793096.

[2]. Wong W. Idiopathic Nephrotic syndrome in New Zealand children, demographic, clinical features, initial management and outcome after twelve-month follow up results of a three year national surveillance study. J Pediatr Child Health. 2007; 43:337-341. PMID: 17489822 .

[3]. Tarshish P, Tobin JN, Bernstein J, Edelmann CM Jr. Prognostic significance of the early course of minimal change Nephrotic syndrome: report of the International Study of Kidney Disease in Children. J Am Soc Nephrol. 1997; 8:769-776. PMID: 9176846.

[4]. Bagga A, Mantan M. Nephrotic syndrome in children. Indian J Med Res. 2005; 122:13-28. PMID: 16106086.

[5]. Yu L, Wen ZY, Hua SD, et al. Analysis of clinical characteristics in infantile nephritic syndrome. Chinese Journal of Practical Pediatrics. 2002; 17:553-555. PMID: 3178149.

[6]. Hodson EM, Willis NS, Craig JC. Non-corticosteroid treatment for Nephrotic syndrome in children. Cochrane Database of Systematic Reviews. 2008; 23(1). Article ID CD002290. PMID: 18254005.

[7]. Yorgin PD, Krasher J, Al-Uzri AY. Pulse methylprednisolone treatment of idiopathic steroid-resistant Nephrotic syndrome. Pediatric Nephrology. 2001; 16(3):245-250. PMID: 11322372.

[8]. Segarra A, Vila J, Pou L. Combined therapy of tacrolimus and corticosteroids in cyclosporine-resistant or -dependent idiopathic focal glomerulosclerosis: a preliminary uncontrolled study with prospective follow-up. Nephrology Dialysis Transplantation. 2002; 17(4):655-662. PMID: 11917061.

[9]. Ehrich JH, Geerlings C, Zivicnjak M, Franke D, Geerlings H, Gellermann J. Steroid-resistant idiopathic childhood nephrosis: over diagnosed and undertreated. Nephrology Dialysis Transplantation. 2007; 22(8):2183-2193. PMID: 17504846.

[10]. Ejaz I, Khan HI, Javaid BK, Rasool G, Bhatti MT. Histopathological diagnosis and outcome of pediatric Nephrotic syndrome. Journal of the College of Physicians and Surgeons Pakistan. 2004; 14(4):229-233. PMID: 15228828.

[11]. Otukesh H, Otukesh S, Mojtahedzadeh M, Hoseini R et al; Management and outcome of steroid-resistant Nephrotic syndrome in children; Iran J Kidney Dis. 2009 Oct;3(4):210-217. PMID: 1984152.

[12]. Segar Hodson EM, Habashy D, Craig JC. Interventions for idiopathic steroid-resistant Nephrotic syndrome in children. Cochrane Database Syst Rev. 2006 Apr 19; Volume 2. Article: CD003594. PMID: 16625586.

[13]. Olowu WA, Adelusola KA, Adefehinti O. Childhood idiopathic steroid resistant Nephrotic syndrome in Southwestern Nigeria. Saudi J Kidney Dis Transpl 2010; 21:979-990. PMID: 20814150. 
[14]. Bagga A, Gulati A, Gulati S, Mehta K.P, Vijayakumar M. Management of steroid resistant Nephrotic syndrome. Indian Pediatrics 2009; 46:35-47. PMID: 19179716.

[15]. Hamasaki Y, Yoshikawa N, Hattori S, Sasaki S et al. Cyclosporine and steroid therapy in children with steroid-resistant Nephrotic syndrome, Japanese Study Group of Renal Disease. Pediatr Nephrol. 2009 Nov; 24(11):2177-2185. PMID: 19714370.

[16]. Habashy D, Hodson EM, Craig JC. Interventions for steroid-resistant Nephrotic syndrome: a systematic review. Pediatr Nephrol. 2003; 18(9):906-912. Epub 2003 Jun 26. PMID: 12836096.

[17]. Gulati S, Sengupta D, Sharma RK, Sharma A, Gupta RK, Singh U et al. Steroid resistant Nephrotic syndrome: role of histopathology. Indian Pediatr. 2006 Jan; 43(1):55-60. PMID: 16465008.

[18]. Kari JA, Halawani M. Treatment of steroid resistant Nephrotic syndrome in children. Saudi J Kidney Dis Transpl. 2010 May; 21(3):484-487. PMID: 20427873.

[19]. Gulati S, Prasad N, Sharma RK, Kumar A, Gupta A, Baburaj VP. Tacrolimus: a new therapy for steroid-resistant Nephrotic syndrome in children. Nephrol Dial Transplant. 2008 Mar; 23(3):910-913. Epub 2007 Nov 26. PMID: 18039644.

[20]. Gargah TT, Lakhoua MR. Mycophenolate moefetil in treatment of childhood steroid-resistant Nephrotic syndrome. J Nephrol. 2011 Mar-Apr; 24(2):203-207. PMID: 21319130.

[21]. Gargah T, Labassi A, Goucha-Louzir R, Ben Moussa F, Lakhoua MR. Histopathological spectrum of childhood idiopathic steroidresistant Nephrotic syndrome in Tunisia. Tunis Med. 2011 Mar; 89(3): 258-61. PMID: 21387228. 